\title{
TOWARDS IMPROVEMENT OF SIGNAL'S RANGE-DOPPLER CHARACTERISTICS
}

\author{
I. Gladkova*, D. Chebanov ${ }^{\dagger}$ \\ * City College of New York, 138th Street and Convent Avenue, New York, NY 10031, USA \\ e-mail: gladkova@cs.ccny.cuny.edu, fax: (212)-650-6248 \\ $\dagger$ Graduate Center of CUNY, 365 Fifth Avenue, New York, NY 10016, USA \\ e-mail: chebanov@cs.ccny.cuny.edu
}

Keywords: radar waveform design, ambiguity function, prolate spheroidal wave functions, nonlinear optimization.

\begin{abstract}
In this paper, we extend our modification of the Wilcox waveform approximation technique [8] to the case of prolate spheroidal wave functions. We have previously carried out a corresponding analysis in the case of Hermite waveforms [3, 4].
\end{abstract}

\section{Introduction}

In [8], Wilcox introduced a general method using Hilbert space technique, for finding a waveform whose ambiguity function is a good approximation over all of $R^{2}$ to a specified function. In previous papers $[3,4]$ we have adapted Wilcox's method to the problem of producing a close match, over a specified subregion of $R^{2}$, to a good ambiguity surface, and taking a specific orthonormal basis (Hermite waveforms) as the starting point.

The optimization over a subregion of $R^{2}$ generalizes Wicox's approach, which optimizes over all of $R^{2}$. There are new subtleties that appear with this approach, since we can seek, for example, to make an ambiguity small over some region, which, if successful, will push the bulk of the function outside the region where we want it to be small. Obviously, this is not possible if the region is all of $R^{2}$, because of the volume property of the ambiguity function.

An obvious long range project is an investigation of the relationship between the choice of an orthonormal basis and the desired region $G \in R^{2}$. For now, we consider it worthwhile to look at what happens with specific examples of the orthonormal basis and region $G$.

As noted before, we have recently carried out such a project for Hermite waveforms and $G$ having circular symmetry, which is a natural property for the $G$ associated to an Hermite basis. In the present paper, we will conduct a similar investigation for the family of prolate spheroidal wave functions. As we will see, in this case the natural geometry of the regions $G$ differs from the circular symmetry of the Hermite case.

To clarify our exposition, we will next give definitions needed in this work and recall the technique for producing waveforms that match a specified ambiguity function that was first introduced by Wilcox in [8].

\section{Construction of ambiguity surfaces with de- sired characteristics}

The ambiguity function of a waveform $u(t)$, which is assumed to be either a real or complex valued function of time with unit energy, is defined by

$$
\chi_{u}(\tau, \nu)=\int_{-\infty}^{\infty} u\left(t-\frac{\tau}{2}\right) \overline{u\left(t+\frac{\tau}{2}\right)} e^{-j 2 \pi \nu t} d t .
$$

The ambiguity function has a tidy set of interlocking properties (see $[1,2,9]$ for details) that strongly constrain the set of twodimensional functions that can be ambiguity functions. For example, the volume under the ambiguity surface, $|\chi(\tau, \nu)|$, must equal the square of its maximum achieved at the origin. Any attempt to push down the ambiguity surface in one place makes it pop up somewhere else [1].

It is well-known fact that an ideal radar waveform would produce an ambiguity surface that is zero everywhere except the origin. Such a function would have ideal range-doppler characteristics. However, no finite energy signal gives rise to that surface [2]. Nevertheless there might be a waveform with ambiguity surface that is an acceptable approximation to the desired surface. In [8], Wilcox laid down a foundation of the theory that allows the approximation of an arbitrary square-integrable function by an ambiguity function over $R^{2}$. In practice, however, engineers have a general idea of acceptable shape rather than the formulas describing it, thus making Wilcox's algorithm not applicable. In [3, 4], we have demonstrated that the modification of the Wilcox's general idea can lead to good results if we restrict the attention to a suitably specified subset $G$ of $R^{2}$, rather than all of $R^{2}$. Below we briefly recall the main steps of our approach. 
Since any waveform $u(t)$ under consideration is a squareintegrable function of time (that is $u(t) \in L_{R}^{2}$ ), it can be represented, by the Riesz-Fischer theorem [6], as

$$
u(t)=\lim _{N \rightarrow \infty} \sum_{m=0}^{N} a_{m} \phi_{m}(t),
$$

where $a_{m}=<u, \phi_{m}>_{L_{R}^{2}}, \lim _{N \rightarrow \infty} \sum_{m=0}^{N}\left|a_{m}\right|^{2}=1$, and the sequence

$$
\phi_{0}(t), \quad \phi_{1}(t), \quad \ldots, \quad \phi_{m}(t), \quad \ldots
$$

constitutes an orthonormal basis in $L_{R}^{2}$. In the above formulas and hereafter, we use the following notations:

$$
<g, h>_{L_{E}^{2}}=\int_{E} g \bar{h} d E, \quad\|g\|_{L_{E}^{2}}^{2}=<g, g>_{L_{E}^{2}},
$$

where $g, h \in L_{R^{k}}^{2}$ and $E \subseteq R^{k}$ for some natural $k$.

Assuming that some orthonormal basis (2) is fixed, our further consideration will be related to the sequence of classes $V_{N}(N=0,1, \ldots)$ defined as follows

Definition. A function $u(t)$ is in class $V_{N}(N=$ $0,1, \ldots) \Longleftrightarrow$

$$
u(t)=\sum_{m=0}^{N} a_{m} \phi_{m}(t)
$$

such that $a_{m}=<u, \phi_{m}>_{L_{R}^{2}}$ and $a_{m} \in S_{N}$, where $S_{N}$ is the $N$-dimensional unit sphere: $\sum_{m=0}^{N}\left|a_{m}\right|^{2}=1$.

Now, we can formulate the problem of finding $u \in V_{N}$ whose ambiguity function $\chi_{u}(\tau, \nu)$ is close to $F(\tau, \nu)$ as in [3]:

Assume that some orthonormal basis (2) and some region $G \subseteq R^{2}$ are fixed. Then, a given function $F \in L_{R^{2}}^{2}$ and $a$ positive integer $N$, find

$$
\begin{aligned}
& \underset{u \in V_{N}}{\arg \min }\left\|F-\chi_{u}(\tau, \nu)\right\|_{L_{G}^{2}}^{2} \\
& =\underset{u \in V_{N}}{\arg \min }\left(\left\|\chi_{u}(\tau, \nu)\right\|_{L_{G}^{2}}^{2}\right. \\
& \left.\quad-2 \Re\left\{<F, \chi_{u}(\tau, \nu)>_{L_{G}^{2}}\right\}\right)+\|F\|_{L_{G}^{2}}^{2} .
\end{aligned}
$$

From a practical point of view, it is desired to construct waveforms producing surfaces which are very small everywhere in some (perhaps, quite large) neighborhood of the origin and have a peak at that point. This explain the choice of $F(\tau, \nu)$ to be equal to zero in the chosen region and can be thought as an approximation of the ideal ambiguity surface over the region of interest. Based on this observation, we have formulated [3, 4] the following modification of problem (4) which is the subject of study of this paper:
Find a waveform $u(t) \in V_{N}$ such that its ambiguity surface $\left|\chi_{u}(\tau, \nu)\right|$ is the best approximation to the ideal ambiguity function in the mean square sense over some bounded region $G$ containing the origin, i.e.

$$
\underset{u \in V_{N}}{\arg \min }\left\|\chi_{u}\right\|_{L_{G}^{2}}^{2}
$$

It should be noted here that the solution(s) of the non-linear problem (5) significantly depends on the choice of region $G$ as well as basis functions $\left\{\phi_{k}(t)\right\}$. In [3, 4], we have demonstrated the performance of our approach for the case when $G$ is a circular region surrounding the origin and $\left\{\phi_{k}(t)\right\}$ are Hermite waveforms $[5,8]$. In this paper we deal with another orthonormal basis which is known in the literature as the most energy concentrated basis in the space of bandlimited signals.

\section{Prolate spheroidal functions}

We now, as indicated earlier in this paper, specialize to the case where the orthonormal basis (2) consists of prolate spheroidal wave functions. The elegant standard reference for these functions is [7], from which we now recall their definition and a few basic properties.

The prolate spheroidals arise from an optimization problem:

$$
\operatorname{maximize} \frac{\int_{-T / 2}^{T / 2} u^{2}(t) d t}{\int_{-\infty}^{\infty} u^{2}(t) d t}
$$

for all functions in $L_{R}^{2}$ whose amplitude spectra vanish for $|\nu|>W$, i.e. in the space of bandlimited signals $B_{W}$.

Solutions (prolate spheroidal wave functions) of (6) satisfy an integral equation with kernel $\sin \left(\pi W T\left(t-t^{\prime}\right)\right) /\left(\pi\left(t-t^{\prime}\right)\right)$, i.e.

$$
\int_{-1}^{1} \frac{\sin \left(\pi W T\left(t-t^{\prime}\right)\right)}{\pi\left(t-t^{\prime}\right)} \psi\left(t^{\prime}\right) d t^{\prime}=\lambda \psi(t), \quad|t|>1
$$

and also satisfy a second order linear ordinary differential equation

$$
\frac{d}{d t}\left(1-t^{2}\right) \frac{d \psi}{d t}+\left(\chi-(\pi W T)^{2} t^{2}\right) \psi=0
$$

The symmetric kernel of (7) is positive definite, therefore (7) has solutions in $L_{(-1,1)}^{2}$ only for a discrete set of real positive values of $\lambda$, which we will denote $\lambda_{0} \geq \lambda_{1} \geq \lambda_{2} \geq \cdots$, with the corresponding eigenfunctions $\psi_{0}(t), \psi_{1}(t), \psi_{2}(t), \cdots$ that can be chosen to be real and orthogonal on $(-1,1)$. They are also complete in $L_{(-1,1)}^{2}$.

The left hand side of (7) is well defined for all $t \in R$, so $\psi_{n}(t)$ can be defined on $R$ and normalized to unit energy there. Then $\lambda_{n}$ is the fraction of the energy of $\psi_{n}$ that lies in the interval $(-1,1)$.

Prolate spheroidal wave functions have many remarkable properties and can provide a very useful set of bandlimited signals that can be defined as follows [7]: 
Suppose $W>0$ and $T>0$ are given. Define

$$
\phi_{n}(t)=\sqrt{\frac{2}{T}} \psi_{n}\left(\frac{2 t}{T}\right) .
$$

Then

- $\phi_{n}(t) \in B_{W}$,

- $\int_{-T / 2}^{T / 2} \phi_{n}(t) \phi_{m}(t) d t=\lambda_{n} \delta_{m n}$,

- $\int_{-\infty}^{\infty} \phi_{n}(t) \phi_{m}(t) d t=\delta_{m n}$

- the $\phi_{n}(t)$ are complete in $B_{W}$ for $t \in R$

- the $\phi_{n}(t)$ are complete in $L_{(-T / 2, T / 2)}^{2}$.

- among signals in $B_{W}, \phi_{n}(t)$ is the most concentrated signal that is orthogonal to $\phi_{0}(t), \phi_{1}(t), \ldots, \phi_{n-1}(t)$

Figure 1 illustrates $\phi_{20}(t), \phi_{100}(t), \phi_{180}(t)$, and $\phi_{360}(t)$. The partial diagram of the ambiguity surface of $\phi_{20}(t)$ is depicted in Figure 2.
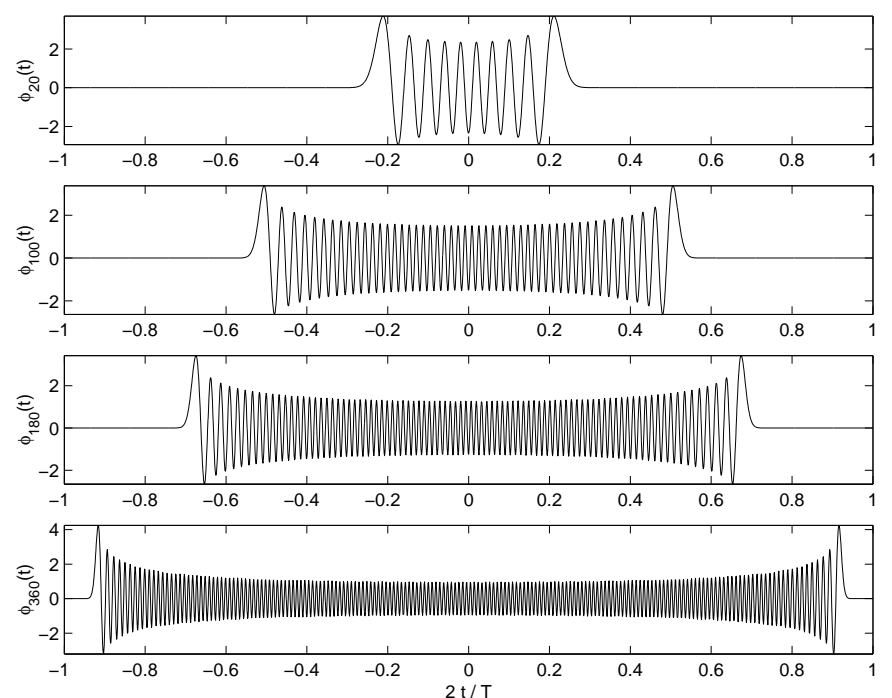

Figure 1: $\phi_{n}(t)$ for $n=20, n=100, n=180$, and $n=360$.

\section{Numerical results}

In what follows, our calculations with prolate spheroidal wave functions will be carried using Matlab's Signal Processing Toolbox program dpss. Next we will consider a few numerical solutions of the minimization problem (5) for some special cases of region $G$. We will start with a circular ring $G=\left\{(\tau, \nu): r^{2} \leq \tau^{2}+\nu^{2} \leq R^{2}\right\}$ surrounding the main lobe that was considered in [3]. A relevant part of the ambiguity surface is shown in figure 3 . The height of the sidelobe peaks in the region of interest appears to be less than $-19 \mathrm{~dB}$ which is by $11 \mathrm{~dB}$ higher than the sidelobe levels in the same region for the solution obtained via approximation by Hermite basis.

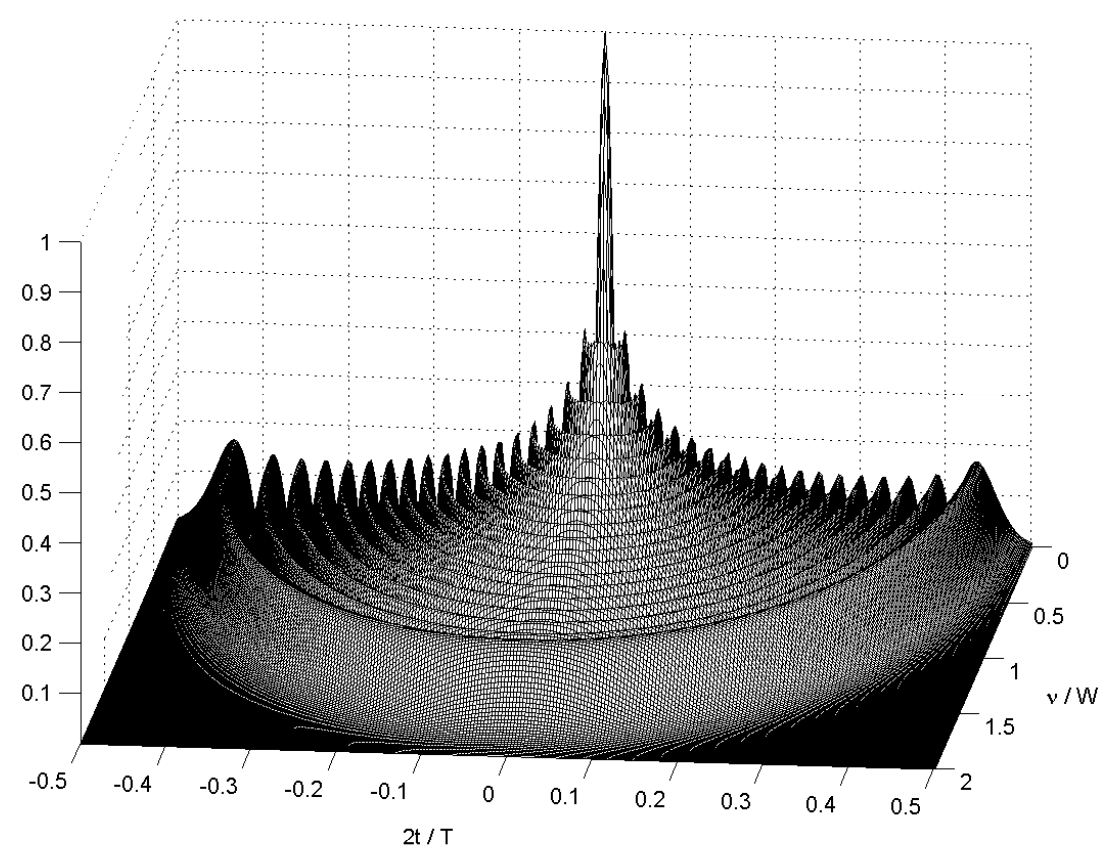

Figure 2: Partial ambiguity surface of $\phi_{20}(t)$. 


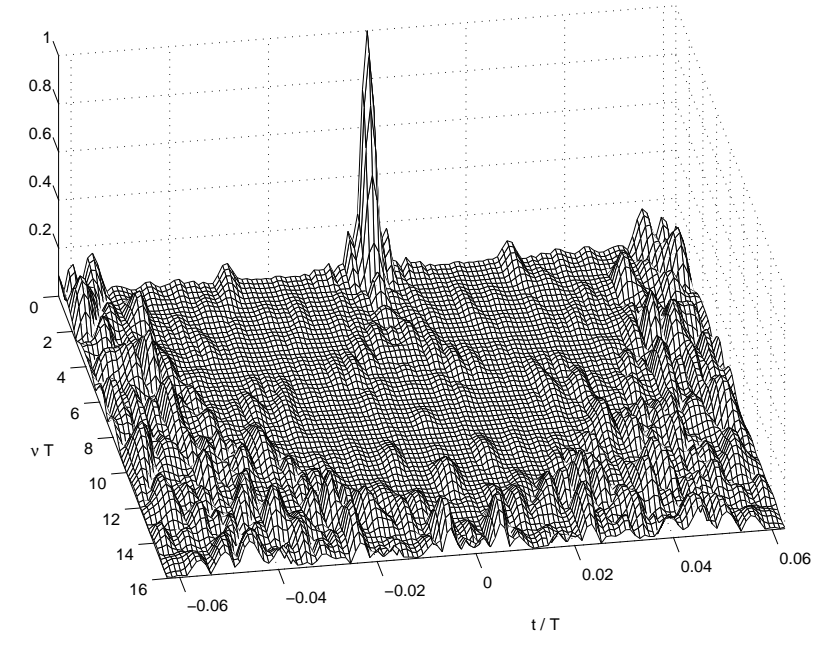

Figure 3: Partial ambiguity surface with suppressed sidelobes in the circular ring.

Figure 4 displays part of the ambiguity surface for Hermite basis. As can be noted, the width of the main lobe is bigger in figure 4, since the number (200 fn's) of the basis functions used in the case of Hermite waveforms was less than in the case of prolate shperoidals (400 fn's).

As it is evident from the figures 3 and 4 , the solution of the minimization problem (5) depends on the choice of the basis $\left\{\phi_{n}\right\}$ and the region $G$. While the Hermite waveforms seem to be a good match to regions with circular symmetries, prolate spheroidal waveforms do not seem to perform well there.

We will next consider the example when the region $G$ is a rectangle $[-T, T] \times[-1 / T, 1 / T]$, i.e. a strip along the time cross-

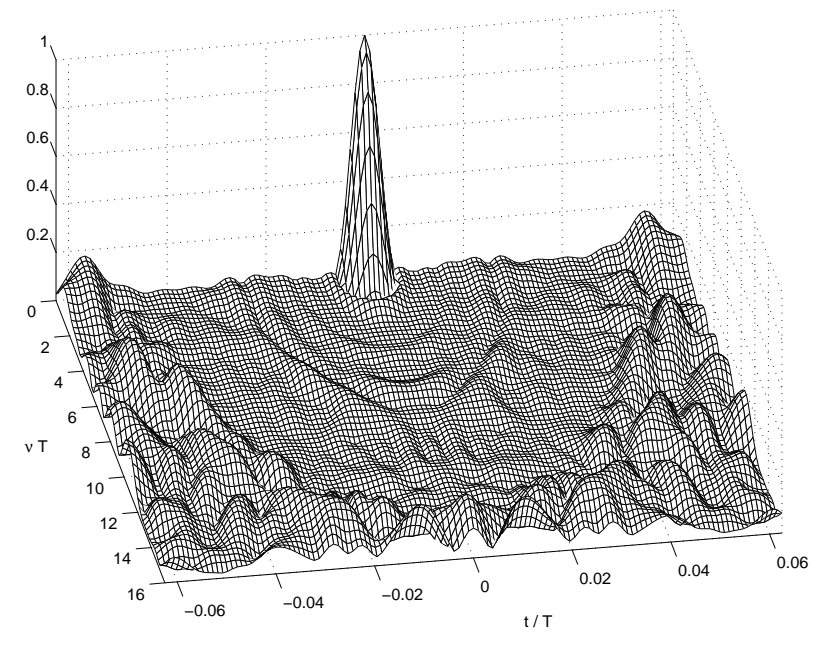

Figure 4: Partial ambiguity surface with suppressed sidelobes in the circular ring over Hermite basis as in [3]

section. Figure 5 illustrates the ambiguity surface in the part of $G$ that contains the main lobe. The cross-section in logarithmic scale is depicted in figure 6 . All sidelobes are below $-40 \mathrm{~dB}$.

We should note that the waveforms obtained via the procedure described in this paper do not have to satisfy the usual desideratum of constant amplitude. Simply forcing the obtained waveform to have a constant envelope causes a degradation of the ambiguity profile.

There are various modifications of (5) that address this issue. For example, one can add a penalty term like $\mu \int_{-T / 2}^{T / 2}\left(\frac{d}{d t}(u(t) \overline{u(t)})\right)^{2} d t$ that will prevent the amplitude of

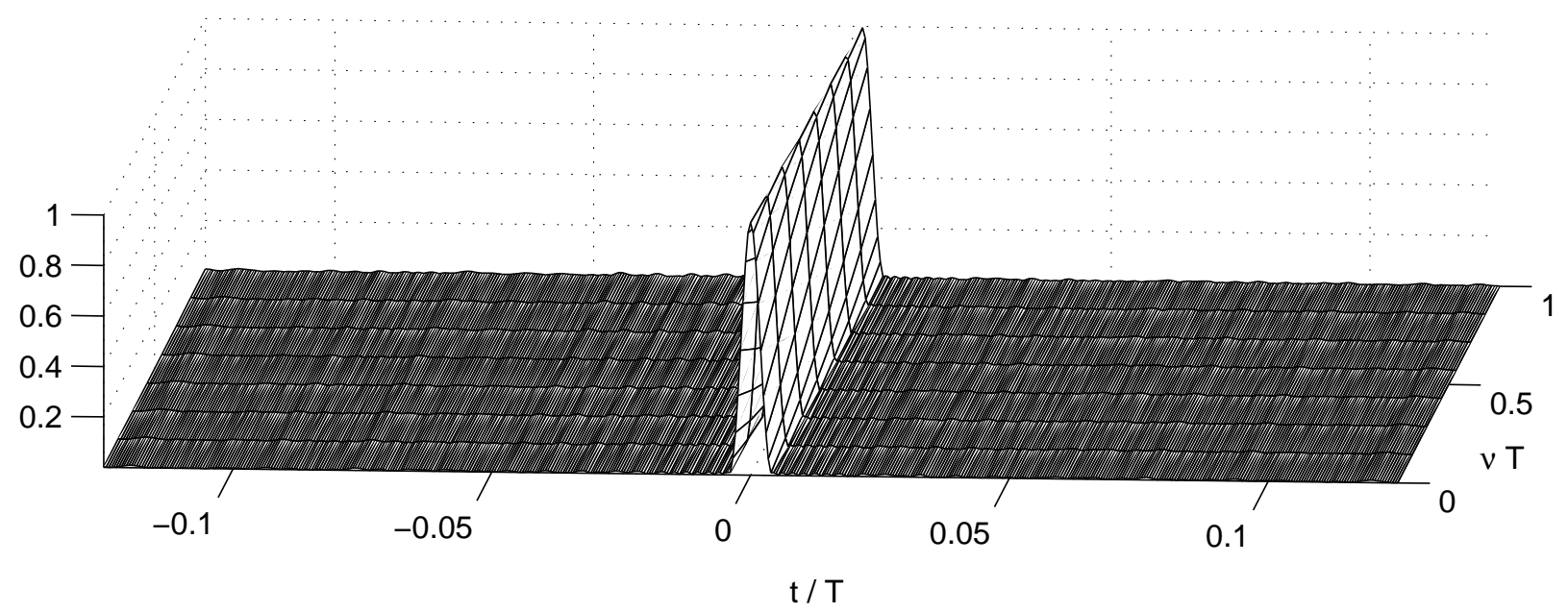

Figure 5: Partial ambiguity surface with suppressed sidelobes in the rectangular region $G$ containing time-delay axis. 
$u(t)$ from changing too much. The result of such a modification is shown below. Figure 7 illustrates the autocorrelation function in logarithmic scale. The sidelobes are higher than in figure 6 by $8 \mathrm{~dB}$.

\section{Conclusion}

In this paper we discuss the construction of waveforms with optimal ambiguity characteristics in a chosen a priori region containing the main lobe. The suggested approach is based on the projection of the signal onto an appropriate orthonormal basis and approximating the signal with desired ambiguity properties by a finite number of basis waveforms. We have considered prolate spheroidal waveforms as the basis functions and discussed the problem of minimizing the volume under the ambiguity surface over two type of regions: a circular ring and a rectangular strip containing the time-delay axis.

\section{Acknowledgments}

This work is being sponsored in part by the DOD Army Research Office under Contract DAAD19-03-1-0329

\section{References}

[1] R.E. Blahut. "Theory of Remote Surveillance Algorithms", in "Radar and Sonar", Part 1, Springer-Verlag, (1991).

[2] C.E. Cook, M. Bernfeld, Radar Signal: An Introduction to Theory and Applications, Academic Press Inc., (1967).

[3] I. Gladkova, D. Chebanov. "On a new extension of Wilcox's method", WSEAS Trans. on Mathematics, volume 3, pp. 244-249, (2004).

[4] I. Gladkova, D. Chebanov. "On the synthesis problem for a waveform having a nearly ideal ambiguity surface", Proceedings of the International Conference RADAR 2004, Toulouse, France, (2004).

[5] J.R. Klauder. "The design of radar signals having both high range resolution and high velocity resolution", Bell System Tech. J., volume 39, no. 4, pp. 809-820, (1960).

[6] F. Riesz, B. Sz.-Nagy. ”Functional Analysis”, F. Ungar Publishing Co., (1955).

[7] D. Slepian. "Some comments of Fourier analysis, uncertainty and modeling", SIAM Review, volume 25, no. 3, pp. 379-393, (1983).

[8] C.H. Wilcox. "The synthesis problem for radar ambiguity functions", MRC Tech. Summary Report 157, US Army, Univ. of Wisconsin, Madison, Wisconsin, (1960).

[9] P.M. Woodward. "Probability and Information Theory, with Applications to Radar”, Pergamon Press, (1953).
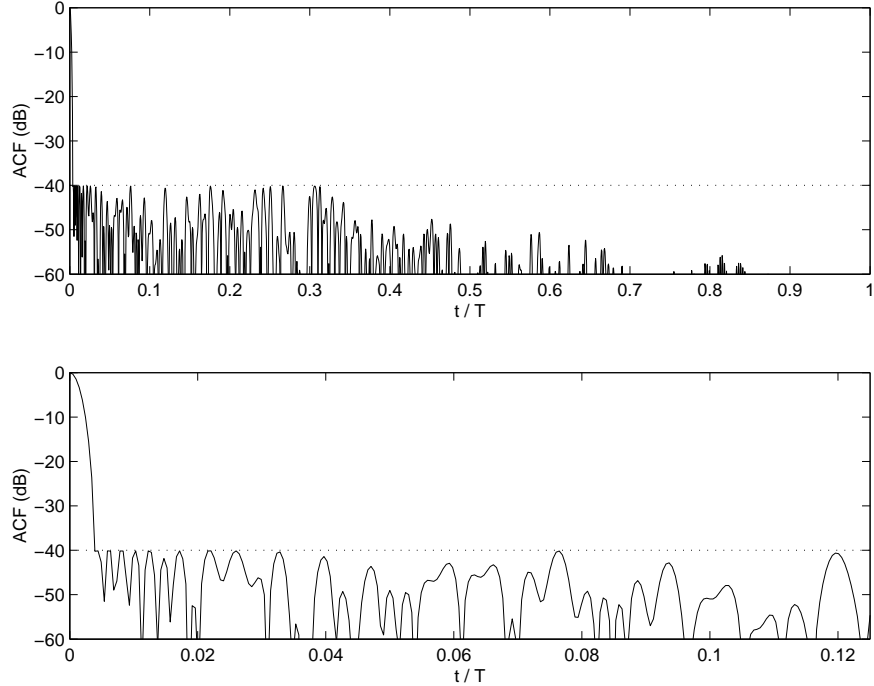

Figure 6: Autocorrelation function (in $\mathrm{dB}$ ) for $0<\tau<T$ (top) and $0<\tau<T / 16$ (bottom)
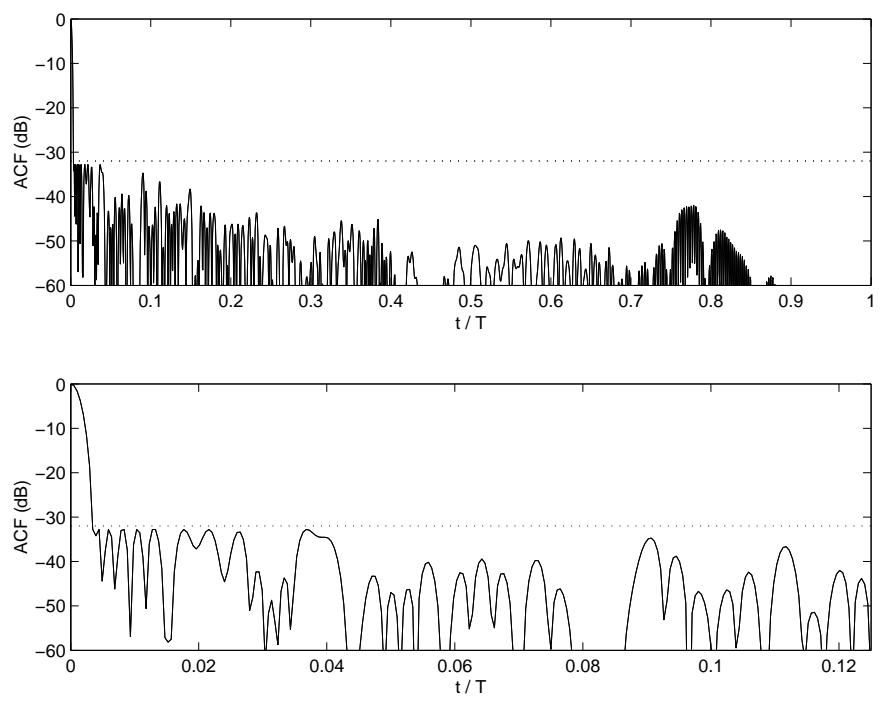

Figure 7: Autocorrelation function (in $\mathrm{dB}$ ) for $0<\tau<T$ (top) and $0<\tau<T / 16$ (bottom)
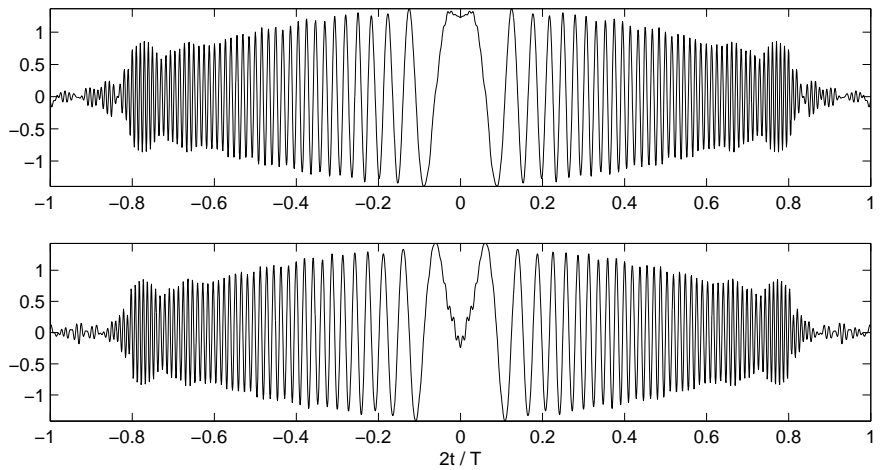

Figure 8: Real (top) and imaginary (bottom) parts of the corresponding waveform. 